\title{
A Humean Guide to Spielraum Probabilities
}

\author{
Claus Beisbart \\ Institut für Philosophie \\ Universität Bern \\ Länggassstr. 49a \\ CH-3000 Bern 9 \\ Switzerland \\ Journal for General Philosophy of Science 47 (2016), \\ pp. 189-216 (special issue edited by J. Rosenthal und C. Seck)
}

July 5, 2016

\begin{abstract}
The most promising accounts of ontic probability include the Spielraum conception of probabilities, which can be traced back to J. v. Kries and H. Poincaré, and the best system account by D. Lewis. This paper aims at comparing both accounts and at combining them to obtain the best of both worlds. The extensions of both Spielraum and best system probabilities do not coincide because the former only apply to systems with a special dynamics. Conversely, Spielraum probabilities may not be part of the best system, e.g. if a broad class of random devices is not often used. Spielraum probabilities have the potential to provide illuminating explanations of frequencies with which outcomes of trials on gambling devices arise. They ultimately fail to account for such frequencies though because they are compatible with frequencies that grossly differ from the values of the respective probabilities. I thus follow recent proposals by M. Strevens and M. Abrams and strengthen the definition of Spielraum probabilities by adding a further condition that restricts some actual frequencies. The resulting account remains limited in scope, but stands objections raised against it in the recent literature: It is neither circular nor based upon arbitrary choices of a measure or of physical variables. Nor does it lack any explanatory power.
\end{abstract}

\section{Introduction}

Some of the deepest results of scientific inquiry come in a probabilistic guise: They do not state what happens, but rather provide the probabilities with which outcomes arise. Quantum mechanics is probabilistic, as is statistical mechanics, and researchers in the special sciences devise all kinds of probabilistic models. One attempt to explain all this 
is to say that nature itself is probabilistic or chancy, that there are ontic probabilities or chances out there in the world, and that probabilistic statements that reflect settled scientific results hold literally true for these chances.

But what are chances, i.e., probabilities that are supposed to exist independently of human knowledge or interests? If some probabilistic statements from science are literally true, what are their truth conditions?

In his "Principien der Wahrscheinlichkeitsrechnung", Johannes von Kries has proposed an account of probabilities (von Kries 1886). Probabilities are defined using socalled Spielräume, i.e., ranges of possibilities (ibid., pp. 24-25). Often, one can measure how large one Spielraum is with respect to another. For instance, if one body can have a speed between 3 and $5 \mathrm{mph}$, and a second a speed between 4 and $5 \mathrm{mph}$, the respective Spielräume have sizes with a ratio of 2:1. Each outcome of an experiment on a gambling device is associated with a Spielraum that realizes the respective outcome. The probability of an outcome then is measured as the relative size of the corresponding Spielraum, compared to that of a suitable reference Spielraum (e.g. ibid., pp. 42-43). Since Spielräume that enter a definition of probability are required to be original (ibid., pp. 35-36), von Kries suggests that Spielräume be defined for initial conditions (ibid., p. 45). In modern terms, the probability of an outcome is measured as the relative size of the region that the initial conditions leading to the outcome occupy. Von Kries further discusses a type of dynamics that yields probabilities the values of which do not much depend on the details of how the initial conditions are distributed (ibid., pp. 49-54). T $^{1}$

Similar ideas as those by von Kries have later been developed and elaborated by Henri Poincaré (Poincaré 1896) and Eberhard Hopf (Hopf 1934; Hopf 1936) and have become known under the name "method of arbitrary functions" ${ }^{2}$ Recent years have seen a number of works that attempt to provide an account of ontic probability by drawing on von Kries and Poincaré (Strevens 2003, Rosenthal 2010, Strevens 2011, Abrams 2012, Myrvold 2012 and Rosenthal 2012). In this paper, I'll discuss a specific proposal of this family. Probabilities that fulfill the proposed definition are called Spielraum probabilities.

In the recent literature, there is another popular account of ontic probability. Lewis (1994) analyzes chances as best system probabilities. The latter are formal probabilities that provide an optimal representation of some respects of the world. Because they obey a principle called Humean supervenience, they are also often called Humean chances $:^{3}$

As Rosenthal (2012), p. 235 points out, "there have to be connections between these [i.e., Spielraum and best system] approaches to objective probability". And indeed, when Frigg \& Hoefer (2010) elaborate Lewis's account, they consider exactly the type of system for which Spielraum probabilities are defined. The aim of my paper is to follow this lead and to relate Kriesean Spielraum probabilities to Humean chances à la

\footnotetext{
${ }^{1}$ For more details on von Kries' view of probabilities see Kamlah (1983) and Heidelberger (2001).

${ }^{2}$ According to von Plato (1983), p. 38, it is not clear whether Poincaré knew von Kries's work when writing Poincaré (1896). See von Plato (1983) for the method of arbitrary functions. For the difference between Spielraum probabilities and the method of arbitrary functions see fn. 13 below.

${ }^{3}$ There are other accounts of ontic probabilities, notably frequentism and various propensity views. These accounts are not very popular though these days. See Gillies (2000) for an introduction to the philosophy of probability and Briggs (2010) for a recent discussion of ontic probabilities.
} 
Lewis. I will compare the respective definitions of ontic probability with their weaknesses and strengths and then scrutinize whether the approaches can be combined to overcome some of the respective difficulties. This will lead to an account that has been proposed by Strevens (2011) and Abrams (2012).

As far as I am aware, the aim of this paper has not yet been pursued in the extant literature. Myrvold (2012) combines Spielraum probabilities with ideas by D. Lewis, but confines himself to Lewis's Principal Principle (Lewis 1980) and does not consider best system probabilities. The most important references for my paper are Frigg \& Hoefer (2010), Strevens (2011), Abrams (2012) and Rosenthal (2012).

The project of this paper is systematic; in particular, I'm not interested in a charitable reconstruction of ideas by von Kries. The focus of this paper is on philosophical issues and not on an appropriate mathematical elaboration. I will thus keep the presentation as informal as possible and gloss over mathematical details.

The paper is organized as follows. Sec. 2 sketches a framework for thinking about ontic probabilities and presents the definitions of Spielraum and best system probabilities. Sec. 3 compares the definitions. Problems of the respective accounts are specified in Sec. 4. In Sec. 5, I combine insights from both accounts in order to solve some of the problems mentioned before. I draw my conclusions in Sec. 6 .

\section{Short guides to Spielraum and best system prob- abilities}

What exactly must be accomplished by a philosophical account of ontic probabilities? And how are chances analyzed following von Kries or Lewis? The aim of this section is to answer these questions.

\subsection{Ontic probabilities}

Simple probabilistic statements assign a real number $r$ from the interval $[0,1]$ to a proposition $q$ as its probability. They take the following form:

PA At time $t$, the probability that $q$ holds true equals $r \in[0,1]$, i.e., $P_{t}(q)=r$.

The probability that $q$ is true has a time index because its value may change even if $q$ refers to an instance of time already. Consider e.g. the proposition that person A finds her way out of a labyrinth by a certain time. The respective probability plausibly depends on A's present location, which in turn varies with time (Lewis 1980, p. 95). For reasons of convenience though, we can often drop time dependence because, as a matter of fact, the values of some probabilities do not depend on time.

On the basis of PA, we can form more complicated probabilistic statements, e.g. conditionals in which PA figures as consequent. Other types of probabilistic statements, e.g. assignments of infinitesimal (Lewis 1980, p. 89) or conditional probabilities may be neglected for the purposes of this paper. Probabilities that are attached no to propositions, but rather to events, can usually be translated in probabilities of propositions (see Howson \& Urbach 2006, p. 14). 
The probability assigned by PA is ontic, if, and only if, the statement is truth-apt, if its truth conditions do not depend on human knowledge or interests and if it is in fact true. Even if there are ontic probabilities, not all probabilistic statements of type PA reflect such probabilities. Some such statements may not even be meant to reflect ontic probabilities, but rather specify epistemic probabilities. Other statements may be supposed to refer to ontic probabilities, but be false, either because the proposition $q$ does not carry an ontic probability at all or because the statement fails to specify the correct value of the ontic probability. There are two things, then, that we would like to know about ontic probabilities: i. Which propositions carry ontic probabilities, i.e., which propositions are chancy? ii. What values do the ontic probabilities take?

Philosophical accounts of ontic probabilities address these questions. Most of them specify a general method to specify the truth conditions for statements about ontic probabilities. The proposed truth conditions for a particular probabilistic claim pick a plurality of possible worlds, and we can then decide whether they are fulfilled in a world. In this paper, we concentrate on reductive accounts of ontic probabilities. Such accounts specify the truth conditions for statements of type PA using only non-probabilistic terms.

Accounts of ontic probabilities are often supposed to provide analyses of what probabilistic statements mean. The accounts discussed in this paper cannot be thought of in this way because the proposed truth conditions are too complicated and technical to capture what people mean to say when they issue probabilistic statements. Nevertheless, the truth conditions provided by an account may entail the conclusions that people typically draw from probabilistic statements. They may pick those facts that people refer to if they speak of probabilities and they may explain what people intend to explain with probabilistic statements. In this paper, accounts of ontic probabilities are understood in this sense.

How can we decide whether such an account of probabilities is successful? Most importantly, every such account has to pick those chances and only those that we take there to be, not just in our world, but also in every other possible world, at least in those close to ours. An account may fail either because it can't be applied to some worlds, because it misidentifies the chancy propositions or because it mistakes the values of chances in some worlds.

The application of this criterion presumes independent commitments as to what chances there are in this and in other worlds. We do have such commitments because scientific findings often come in a probabilistic guise, and we also have intuitions as to what chances are and when they apply.

Nevertheless, both scientific results and intuitions are problematic as independent sources concerning chances. Some probabilistic results from science do not specify chances because the probabilities are only used to encode our own ignorance. The status of the probabilities in other well-respected statements from science, e.g. from statistical mechanics, is a matter of debate.4 Intuitions may be misguided and ill-informed.

\footnotetext{
${ }^{4}$ See Meacham (2010a) and Meacham (2010b) for interpretations of probabilities in statistical physics.
} 
There is no alternative though to using scientific results and intuitions. To avoid problems, we will only draw on uncontroversial ideas about chances.5

How can we compare two reductive accounts of ontic probabilities? Obviously, we can compare their truth conditions. In particular, we can study the mutual logical relationships between them. For instance, the truth conditions of one account may entail those of the other, or vice versa. This is not to be expected though because truth conditions of probabilistic claims pick large pluralities of worlds, and a set of truth conditions only entails another one if the worlds picked by it form a subset of the worlds picked by the other. What is more realistic is that one account covers the other for a subset of worlds, i.e., under additional conditions. This possibility will be investigated later in this paper.

Different statements about ontic probabilities may require different accounts. For instance, probabilistic statements about microevents may require a different account than statements about probabilities of macroevents. I will not assume that one and the same account must apply to all statements about ontic probabilities.

\subsection{A first guide to Spielraum probabilities}

Turn now to our first account, which elaborates ideas by von Kries (1886), Chs. II-III and by others. The proposed truth conditions for probabilistic claims will be said to define Spielraum probabilities (SR probabilities, for short). This is not to prejudge the question of whether Spielraum probabilities are real chances.

Spielraum probabilities attach to propositions that specify the outcomes of trials carried out on what we call chance setups. Many macroscopic gambling devices are such chance setups. To simplify matters, let us focus on coin flips as our example the generalization to other chance setups and other types of systems is straightforward and will be given in SR below.

Each coin flip either yields heads or tails as its outcome. Spielraum probabilities are defined for propositions that specify what the outcome of a particular coin flip is at a time $t_{f}$ at which the coin has just landed.

We assume that each coin flip follows a deterministic dynamics, which is best described in a phase space that is spanned by natural physical characteristics of the coin $x_{j}$ $(j=1, \ldots, N)$. 7 Keller (1986) uses macroscopic characteristics such as the angular velocity of the coin to define a suitable phase space. Each point $\mathbf{x}$ in phase space corresponds to a state of the coin. There is a natural measure over phase space, viz. the Lebesgue measure $\mu$. It assigns real numbers to regions of phase space. The measure is preserved if the dynamics of the system is described using a time-independent Hamiltonian, which we assume is the case 8 Therefore, the measure of a region does not change in time if it is evolved following the dynamics.9

\footnotetext{
${ }^{5}$ For a nuanced view about method in the philosophy of probability see Hájek (1997), pp. 210-211 and Hájek (2010), See also Abrams (2012), p. 346.

6 von Kries (1886), Ch. III considered a "Stoss-Spiel" as defined on his pp. 49-50.

7 See Keller (1986), Diaconis (1998) and Diaconis et al. (2007) for the physics of coin flips.

8 This result is called Liouville's Theorem. See e.g. Arnol'd (1989), Sec. 3.5.

${ }^{9}$ Here we assume realism about phase space. We suppose that each coin flip is properly described
} 
Because the coin has just landed at time $t_{f}$, only some part of phase space, call that region $\Gamma^{t_{f}}$, may be occupied by the system. We assume that the measure of this region, $\mu\left(\Gamma^{t_{f}}\right)$, is finite. The possible outcomes $o_{i}$ of the coin flip induce a partition of $\Gamma^{t_{f}}$ into regions $\Gamma_{i}^{t_{f}}$. For each $i$, the state is in $\Gamma_{i}^{t_{f}}$ at $t_{f}$ if, and only if, the outcome is $o_{i}$.

Granted our assumptions of a time-independent Hamiltonian, the dynamics of the coin can be described using an evolution function $\phi_{T}$, where $T$ is a time interval. If the coin is in state $\mathbf{x}$ at time $t$, it is in state $\phi_{T}(\mathbf{x})$ at time $(t+T)$ and in state $\phi_{-T}(\mathbf{x})$ at time $(t-T)$. For every time interval $T$ the partition $\Gamma_{i}^{t_{f}}$ induces a partition of $\phi_{-T}\left(\Gamma^{t_{f}}\right)$ into sub-regions $\phi_{-T}\left(\Gamma_{i}^{t_{f}}\right)$. Following Rosenthal (2012), p. 225 we call these sub-regions patches 10 Since the measure $\mu$ is preserved in time, we have $\mu\left(\Gamma_{i}^{t_{f}}\right)=\mu\left(\phi_{-T}\left(\Gamma_{i}^{t_{f}}\right)\right)$.

We can define Spielraum probabilities for times $t=t_{f}-T$ for a suitable $T$ if, on top of the assumptions already adopted, the $\phi_{-T}\left(\Gamma_{i}^{t_{f}}\right)$ induce a pattern as you would expect it for a well-mixed dough. Each ingredient of the dough has been spread throughout the dough, and each natural probe you take from the dough has roughly the same composition. For a precise definition, we consider partitions of $\phi_{-T}\left(\Gamma^{t_{f}}\right)$ into connected test regions that each have a very small Lebesgue measure. The test regions are also assumed to have a natural geometry (e.g. of a cube) and a small scale in every direction of phase space. $\phi_{-T}\left(\Gamma_{f}^{t_{f}}\right)$ includes lots of such test regions. If there is a partition such that, for each outcome $o_{i}$ and for each test region $B$ from the partition,

$$
\frac{\mu\left(B \cap \phi_{-T}\left(\Gamma_{i}^{t_{f}}\right)\right)}{\mu(B)}
$$

takes about the same value $r_{i}$, we call the dynamics microconstant with a strike ratio of $r_{i}$ for $o_{i}$ (where we adopt the terminology by Strevens 2003, pp. 52-54). The strike ratio is the relative size of a patch within a test region, as measured by the Lebesgue volume. If there is a constant strike ratio, the SR probability $P_{t_{f}-T}$ that the outcome is $o_{i}$ at $t_{f}$ is identified with the corresponding strike ratio $r_{i}{ }^{11}$

Here then are the general truth conditions for Spielraum probabilities:

SR At time $t=t_{f}-T$, the SR probability that a system produces outcome $o_{i}$ at $t_{f}$ equals $r_{i} \in \mathbb{R}$ if, and only if, the phase-space dynamics follows a stationary Hamiltonian with a transition function $\phi_{T}$ and, at $t_{f}$, the outcomes induce a partition $\Gamma_{j}^{t_{f}}$ that is microconstant with a strike ratio of $r_{i}$ for $o_{i}$. That is, for each test region

using a phase space and that assertions made about this phase space literally pertain to the system itself. This may seem worrisome because most known phase-space descriptions are not literally true due to idealizations. This is not the place though to discuss this worry.

${ }^{10}$ We do not require that the patches are maximally connected, as does Rosenthal.

${ }^{11}$ My definition of SR probabilities is close to the one given by Strevens (2003), p. 128. However, I refer to a specific measure, viz. the Lebesgue measure in my definition, while Strevens does not. This does not much matter for the mathematical elaboration. Rosenthal (2012), p. 226 draws on the Lebesgue too to define SR probabilities, but his definition differs from mine because he is more restrictive about the geometry of the test regions. I assume that such differences about technical details do not matter for the purposes of my discussion. 
$B$ from a suitable partition,

$$
\frac{\mu\left(B \cap \phi_{-T}\left(\Gamma_{i}^{t_{f}}\right)\right)}{\mu(B)} \approx r_{i} .
$$

Usually, SR is fulfilled not just for one instance of time, but during a period of time. However, it is not always fulfilled. At early times $t$, i.e., for $t<t_{f}-T_{\max }$, where $T_{\max }$ is a time interval, the coin is not yet in the air, and the phase space description upon which SR is based does not yet apply. There are thus no SR probabilities yet. For other times $t>t_{f}-T_{\min }$ with a certain time interval $T_{\min }$, the distribution of initial conditions in phase space is not microconstant any more because, in phase space, there are larger connected areas leading to the outcomes $o_{i}$. Accordingly, for times $t>t_{f}-T_{\min }$, there are no SR probabilities any more. This is as it should be because, shortly before the coin lands, it is easily determinable how it will land, and we are not inclined to assign a $P_{t}$ probability at this time any more.

Thus, SR is fulfilled for times $t$ with $t_{f}-T_{\max }<t<t_{f}-T_{\min }$, and we have corresponding SR probabilities $P_{t}{ }^{12}$ For each $t$ in this period, the state of system at this time may be treated as the initial condition relevant for the outcome probability $P_{t}$. Consequently, there is no unique initial time for each coin flip and no need to have one.

SR can yield different probabilities of heads for different coins. This is so if the phase space descriptions differ between the coins, e.g. if differences in the constitution of coins produce different strike ratios. Thus, assuming SR, we can type coins in terms of their chances to land heads.

SR has an important consequence (see Strevens 2003, pp. 55-57 and App. 2.C for a more rigorous statement). Suppose that SR holds for some system at time $\left(t_{f}-T\right)$ and that there is a probability distribution over the initial conditions at that time. Take the meaning of the probability distribution as given. For instance, the distribution may arise from a stochastic dynamics before $\left(t_{f}-T\right)$. Suppose, further, that the probability distribution is well-behaved. This is to say that, with respect to each physical characteristic defining a dimension in phase space, $x_{j}$, the distribution varies smoothly and only insignificantly on scales $\epsilon_{j}$. The deterministic dynamics will then lead to unique outcome probabilities. For each outcome $o_{i}$, we will obtain a probability of $r_{i}$. Whatever the initial probabilities are, if only they are well-behaved, the outcome probabilities are the same. In this sense, the dynamics underlying SR probabilities robustifies input probabilities if there are any 13

Note though that SR does not assume a probability distribution for the initial conditions. If it did so, it would be circular as an analysis of probabilities, and it would rely on other probabilities as an explanation of how ontic probabilities arise. To avoid problems of this type, SR does not assume any probability distribution over initial conditions.

\footnotetext{
${ }^{12}$ Properly speaking, the values of $T_{\min }$ and $T_{\max }$ are specific to a particular coin flip.

13 This result is at the basis of the method of arbitrary functions. Unlike the definition of SR probabilities, the method assumes a probability distribution over initial conditions (von Plato 1983. p. 38).
} 


\subsection{A short guide to Lewis's best system probabilities}

Turn now to Lewis's account of chances and to the definition of best system probabilities (BS probabilities, for short).

This definition is much shaped by the idea of Humean Supervenience. In relation to chances, the latter implies that chances supervene on the so-called Humean mosaic (Lewis 1986b, pp. ix-x): If this mosaic of all fundamental facts is fixed for a world, so are the chancy propositions and the chances. For strict Humeans, fundamental facts concern fundamental particles, their spatio-temporal positions and their intrinsic properties (see Lewis 1986a, pp. ix-x). In particular, fundamental facts are neither probabilistic nor law-like. In the recent literature, many authors do not require Humean supervenience in a strict sense. They only assume supervenience on a quasi-Humean mosaic that precludes probabilities and laws. ${ }^{14}$ They allow that e.g. facts about macroscopic coins are part of the mosaic. In the following, we are only interested in supervenience on a quasi-Humean mosaic.

How then are the chancy propositions identified and the values of the chances determined from the mosaic of a world? According to Lewis, this work is done by the best system of the world. This system is something like an optimal pocket guide of the Universe: very informative, though very simple. It assigns numerical probabilities to some propositions, and these propositions are the chancy propositions, while the respective probabilities are the chances.

Here, systems are sets of sentences, some of which are probabilistic, while others are not. The former assign purely mathematical time-indexed probabilities to propositions or generalize over such probability assignments. Non-probabilistic sentences of each system have to be true for the mosaic.

All systems compete with each other, the standards of the competition being strength, simplicity and fit. Although these standards are decisive for Lewis's account, no precise formal characterization of them has been fixed in the literature, so we can only present them in an informal way. As a consequence, we will have to rely upon intuitive judgments when we apply the standards to concrete systems. In simple cases, a comparison between systems concerning strength or simplicity seems unproblematic indeed.

Strength spells out the idea that the systems be informative. Other things being equal, one system $\mathrm{S}$ is stronger than another, $\mathrm{S}$ ', if its non-probabilistic sentences entail those of S'. Further, other things being equal, $S$ is stronger than $S$ ' if the algebra of chancy propositions of the latter is a sub-algebra of that of $\mathrm{S}^{15}$ Simplicity applies to probabilistic and non-probabilistic sentences alike, the idea being that the systems contain few sentences, only simple formulas and so on. Fit is a demand on probabilistic sentences only. Taken together, the probability assignments of a system define a probability of the conjunction of propositions that are assumed to be chancy. A system has better fit than another if it assigns a higher probability to the conjunction of chancy propositions. Good fit favors probabilities the values of which are close to corresponding

\footnotetext{
${ }^{14}$ See e.g. Frigg \& Hoefer (2010), p. 357.

${ }^{15}$ It is not clear how to combine these pro tanto desiderata in case they conflict; more problems of this kind will follow below.
} 
actual frequencies. Technical problems to define fit are discussed by Elga (2004).

The standards of strength, simplicity and fit can compete with each other because system S may be better than its rival S' regarding one standard, while another standard may favor S' over S. Lewis nevertheless assumes that, in such cases, there is an objective way to decide which system is better overall. And it does seem clear that small costs in, say, simplicity have to be incurred to make a lot of progress on, say, fit.

A system is best if, and only if, it strikes an optimal overall balance between strength, simplicity and fit. If there is a unique system that is best, it defines the chancy propositions and the chances. If there is no unique best system, things are less clear. One may either say that there are no chances because there is no unique best system. Alternatively, the chancy propositions and chances may be claimed to be those on which the best systems agree. Both types of reactions are mentioned in e.g. Lewis (1994), p. 479, albeit not regarding chances, rather than laws. Both options will be taken in account in what follows.

Here then are Lewis's truth conditions for BS probabilities:

BS The BS probability $P_{t}$ of a proposition $q$ is $r$ if, and only if, the best system of the world implies a formal probability of $r$ for $q$ at time $t$.

Here, a system implies a probability assignment if and only if the latter can be derived from the axioms of the system using predicate logic and probability theory.

The following discussions are based upon BS, no further assumptions are presupposed. In particular, it is not assumed that all probabilities in a best system derive from probabilities of micro-physical states.

These then are the accounts. How do they compare to each other and how do they differ?

\section{A short comparison between the accounts}

1. Dynamic nature. The truth conditions for SR probabilities are purely dynamic. That is, whether or not there is an SR probability for a certain proposition at some time $t$ only depends on the deterministic dynamics governing a chance setup. No information enters about the frequencies with which outcomes or initial conditions arise. By contrast, information about the patterns of outcomes or of initial conditions in the real world is important for the question of which BS probabilities there are in a world. If a representational system implies probabilities for a type of outcome, then the frequencies with which this type of outcome arises are used to determine the fit of the system.

2. Compatibility with determinism. There can be SR probabilities in a deterministic world; in fact, the truth conditions for SR probabilities presuppose a deterministic dynamics for the system under consideration. This does not imply that the whole world is deterministic, but SR probabilities are clearly compatible with a deterministic world 16

\footnotetext{
${ }^{16}$ If SR referred to an initial probability distribution, matters would be more complicated and, maybe, different.
} 
By contrast, Lewis (1986c), pp. 117-121 claims BS system probabilities to be incompatible with determinism. But there are good reasons to reject his claim. Lewis (1994), pp. 478, 487 assumes that probabilistic statements in systems yield history-tochance conditionals. Each such conditional specifies a chance $P_{t}(\cdot)$ for time $t$, given the complete history up to $t$ (see Lewis 1980, pp. 95-96). If such history-to-chance conditionals follow from the best system, the dynamics has to be indeterministic ${ }^{17}$ But there is no reason to restrict the probabilistic parts of systems to statements that imply history-to-chance conditionals. We can also allow for probabilistic statements about initial conditions (Loewer 2001, p. 618). Loewer is concerned with the initial conditions for the whole Universe, but we can also admit probabilistic statements about initial conditions for more localized systems. If such statements are part of the best system, there are chances even if the dynamics of a world is fully deterministic. Thus, like SR probabilities, BS probabilities are compatible with determinism. 18

3. Locality. The truth conditions for BS probabilities are global because they refer to patterns that may be anywhere in a world. Consequently, local information about a system does never suffice to justify claims about certain BS probabilities. By contrast, the truth conditions for SR probabilities only refer to a specific setup and to processes localized in space and time. Only if we assume that, what laws there are, depends on processes elsewhere, the truth conditions for SR probabilities refer to patterns elsewhere in the world.

4. Humean supervenience. BS probabilities fulfill Humean supervenience because they are identified using a best system of a world. If two worlds do not differ in their mosaics, neither do their respective best systems nor their chances. Whether or not SR probabilities respect Humean supervenience turns on additional assumptions. To see this, suppose, for the sake of hypothesis, that two worlds do not differ in their mosaics, but that they have different SR probabilities for coin flips. This can only be so if the relevant phase space descriptions of the coin flips differ. The phase space description is determined by the laws and contingent facts about the coin and the conditions under which it is flipped. The latter cannot differ between both worlds if their mosaics are identical. Thus, the dynamical laws must differ. This can only be so if dynamical laws do not respect supervenience. Thus, SR probabilities can only violate Humean supervenience if some laws do. Note that the converse is not true: If the pertinent laws violate Humean supervenience, the SR probabilities may nevertheless not do so because microconstancy and the strike ratio are not affected ${ }^{19}$

\footnotetext{
17 Of course, formally, a system may describe a deterministic dynamics using probabilities that only take the values 0 or 1 . But a best system cannot do so because such probabilities make the description of a deterministic dynamics more complicated than necessary.

18 As Hoefer (2007), pp. 558-560 points out, Lewis may have inferred the incompatibility between determinism and the existence of chances from a version of the Principal Principle, but Hoefer suggests that this very version is problematic. See Frigg \& Hoefer (2010) for more discussion.

${ }^{19}$ It may be objected that one and the same mosaic is compatible with different SR probabilities because the same coin flip may be described using different phase spaces and because SR probabilities depend on the phase space chosen. However, in this paper, we assume that there is a literally true phase space description of the system (see fn. 9p). If there are several true phase space descriptions, we expect them to agree upon SR probabilities.
} 
As a consequence, SR probabilities are not a problem for Humeans. Humeans such as Lewis (1986a), pp. 11-12 think that the laws of nature supervene on the mosaic. Thus, for them, SR probabilities do so too.

5. Explanation of the statistics of outcomes. Both SR and BS probabilities can explain the statistics of coin flips, at least if additional assumptions are adopted; but explanations in terms of SR probabilities run deeper 20 To see this, note first the following observations about coin flips.

E1 Outcomes of individual coin flips are difficult to predict because they do not well correlate with other natural characteristics of coin flips that are typically known or controlled at the usual level of accuracy. Because of the lack of good predictors, the patterns of outcomes may be called irregular or random looking.

E2 We mostly observe certain stable frequencies for the outcomes of coin flips.

Every explanation of E1 or E2 in terms of ontic probabilities starts from the assumption that coins have a certain chance of landing heads. Different accounts of chances unpack this statement in different ways and then draw consequences that eventually lead to E1 and E2.

Using SR, E1 can be explained as follows: Due to microconstancy, the initial conditions that lead to a specific outcome are not concentrated in some part of phase space. Rather, for each possible outcome, each test region in phase space contains points that end up with this outcome. Since there is usually no control of, nor knowledge about, the scales smaller than those of the test regions, the outcomes escape control and knowledge too. E2 can also be explained using SR: Due to the deterministic dynamics, the statistics of outcomes coincides with the statistics of patches at an initial time. Consider a specific series of coin flips. It is very natural to expect that the relative frequency of initial conditions that are within a patch and thus the relative frequency of corresponding outcomes is roughly the same as the relative Lebesgue measure of this patch and thus the strike ratio. This expectation is particularly natural since the geometry of the patches is such that every small test region contains the same relative Lebesgue measure of the patch. Obtaining a frequency of heads that considerably differs from the corresponding strike ratio would require some fine-tuning of the initial conditions. Since we do not normally expect such a fine-tuning, we expect that the frequencies of the outcomes approximate the corresponding strike ratios.

These explanations of E1 and E2 run fairly deep because they draw on the dynamics of coin flips. But admittedly, the explanation of E2 is based upon an additional assumption (see p. 23 below).

BS probabilities too can explain the characteristic features observed in patterns of coin flips. Consider first E1. Since there are BS probabilities of outcomes of coin flips, the best system implies probabilities of these outcomes. This means that the outcomes cannot be derived from a simple system of deterministic laws and additional information part of the best system. In this sense, the prediction of the outcomes of coin flips is

\footnotetext{
${ }^{20}$ See Rosenthal (2012), pp. 223-224, and pp. 226-227 for the explanatory power of SR probabilities and ibid., p. 219 and Abrams (2012), p. 245 for the way in which BS probabilities explain.
} 
not entirely straight-forward. However, BS does not imply that the results of coin flips cannot be easily predicted using additional information that is not part of the best system. Further, the explanation of why prediction is difficult does not provide any insight into why outcomes of coin flips are difficult to predict.

We can use BS to explain E2 as follows: In a world like ours, the best system contains generalizations about probabilities for coin flips. These generalizations allow us to derive chances for single outcomes and for finite series of outcomes. Since the best system provides good fit, the frequencies with which the outcomes arise have to take values close to those of the probabilities. If the generalizations about probabilities count as laws, as Lewis (1994), p. 480 suggests, the explanation just quoted draws on probabilistic laws.

This explanation of E2 seems shallower than that in terms of SR probabilities because the alleged probabilistic laws are just sentences in the best system and thus do no more than summarizing features of patterns within the mosaic 212 How shallow the explanation is depends on how exactly the chances of outcomes of coin flips are derived from the best system. If the best system contains an axiom about the probabilities with which outcomes of coin flips (and, maybe, similar random devices) arise, the explanation given by BS probabilities is fairly shallow because the general statements from which the chances of the outcomes are derived do not do more than describe the patterns with which the outcomes arise as a matter of fact. Alternatively, chances of outcomes of coin flips may in principle be derived from chances about microscopic events. This is not a plausible suggestion though because there is no simple way to provide probabilities that apply to microscopic events and that explain the probabilities with which the outcomes of coin flips arise. Finally, the chances of outcomes of coin flips may in principle derive from probabilities of initial conditions for coin flips. Whether this is so depends on the world (see p. 16 below).

6. Extension. For a final discussion point, consider the question of whether the truth conditions of one account imply those of the other, or vice versa, at least for some types of worlds?

There are probabilities that are clearly covered by BS, but not by SR. Quantummechanical probabilities in our world are very plausibly part of the best system (Lewis 1994 uses probabilities for radioactive decay as his favorite example), but they do not arise from the type of dynamics that is characterized in SR. The point generalizes to all probabilities that are used to describe a fundamental stochastic dynamics. So in some worlds (including our own), BS picks chances that are not recognized by SR.

Conversely, are SR probabilities always BS probabilities? This question is touched upon by Hoefer (2007), p. 564 who submits that gambling devices produce Humean probabilities (see also ibid., pp. 568-569). Since the outcomes of trials on many gambling devices have SR probabilities, this suggests that SR probabilities form one possible realization of BS probabilities. Likewise, Frigg \& Hoefer (2010), pp. 358-361 argue that

21 This point is raised by Rosenthal (2012), p. 219. In this passage, Rosenthal refers to frequentist views, and his point is particularly convincing for actual frequentism as defined by Hájek (1997). But for Rosenthal, Lewis's best system account of chances is a sort of frequentism (ibid., p. 218), so the criticism should carry to Lewisian chances. 
the best system of our world assigns a probability of .5 to coins landing heads ${ }^{22}$ But are really all SR probabilities BS probabilities, and are they so in all worlds?

To answer this question, we consider a certain type of coin that has an SR probability of .5 to land heads. Our task is to find out whether the hypothesis

$\mathrm{H}$ Every coin of this type has a probability of .5 to land heads.

follows from the best system.

The answer depends on the world. To see this, note first that, in most worlds, the best system will not contain information (probabilistic or not) about the positions and velocities of micro-particles because such information about contingent matters of fact is too expensive for a best system because the gain in strength is too minimal to justify the costs in simplicity. As a consequence, if the best system implies probabilities for outcomes of coin flips, it does so because it has an axiom that in some way refers to coin flips or to a larger class of trials on random devices (e.g. symmetric bodies with $2,4, \ldots$ faces) ${ }^{23}$.

As a consequence, in some worlds, $\mathrm{H}$ clearly doesn't follow from the best system. To argue this point, I simplify matters and concentrate on worlds in which coins are the only random devices. In these worlds, $\mathrm{H}$ cannot follow from a general statement about all types of random devices.

First, in some worlds, there are only few coin flips. It then will not pay off for a best system to cover information about coin flips at all. The assignment of probabilities to coin flips will produce small gains in strength only, and these gains are outweighed by large costs in simplicity. So we have SR probabilities without counterpart BS probabilities in these worlds.

Second, in some worlds, the patterns of outcomes of coin flips don't look sufficiently random, and the best system succeeds to describe the outcomes using a deterministic rule. For instance, there may be a rule according to which every other coin flip yields heads, if the flips are ordered following cosmic time. This rule is much more informative than $\mathrm{H}$, but not much more complicated. Thus, the best system does not assign probabilities to coin flips. In such a world, we have again SR probabilities without corresponding BS probabilities.

Third, in some worlds, actual frequencies with which the outcomes of coin flips arise do not even roughly match the values of the SR chances. For instance, even though the patches for both outcomes carry about the same Lebesgue measure, the related actual initial conditions are concentrated within one patch (see the next section for more on this). In this case, SR probabilities can't be BS probabilities because the SR probabilities do not produce sufficiently high fit; there may be BS probabilities for coin flips, but their values will not match those of SR probabilities, but rather the frequencies with which the outcomes of coin flips arise.

\footnotetext{
${ }^{22}$ Note though that Hoefer (2007) and Frigg \& Hoefer (2010) consider a slightly modified account of best system probabilities. But their modifications are largely irrelevant for the purposes of this paper.

${ }^{23}$ In a similar way, Frigg \& Hoefer (2010), Sec. 4 argue that $\mathrm{H}$ follows from what they take to be a best system just because the macroscopic patterns of related outcomes call for a simple probabilistic description in terms of $\mathrm{H}$ independently of the microphysics.
} 
The upshot is that there are some worlds in which SR probabilities are not BS probabilities. Only if a world is not in one of the sets of worlds considered so far, can the SR probabilities for coin flips be BS probabilities too. Admittedly though, the three groups of worlds (which have intersections, by the way) are quite far from our world. So what about worlds like ours?

Even here, SR probabilities may not turn out to be BS probabilities. The reason is that the BS account of probabilities runs into problems if there are several types of coins. I will illustrate the problem with unbiased coins and a type of biased coins. While the former have an SR probability of .5 to land heads, the biased coins are stipulated to have a well-defined SR probability of landing heads, but it is different from .5, say, .53. It is of course a substantive question whether there are such biased coins in our world. But we can stipulate that this is so because we are not interested in our world, but rather in worlds that are close to ours. The dynamics of a biased coin should at least be approximately microconstant in our world, so it is not too much of a deviation if we allow for biased coins that have a microconstant dynamics with a strike ratio of .53. There may be other types of biased coins in the world we consider, but whether this is so does not matter for our argument.

How will Humeans handle such worlds? They can try to reproduce the SR probabilities for the different types of coins. To obtain different probabilities of landing heads for different coins, all coin flips must be partitioned into several classes, each of which has its own probability model for the outcomes heads and tails. For instance, one class may contain almost unbiased coins, a second class coins significantly biased in favor of heads. The probability of heads may be .5 in the first class (call this hypothesis $\mathrm{H}_{1}$ ), .53 in the second class $\left(\mathrm{H}_{2}\right)$. Of course, to define the classes, Humeans have to be more precise as to what unbiased coins are, and they cannot define the classes in terms of chances. There are nevertheless several ways to pick the classes, e.g. using the frequencies with which their outcomes arise or using the microconstitution of the coins.

But from a Humean point of view, there is an alternative to the hypotheses $\mathrm{H}_{1}-\mathrm{H}_{2}$, call it H'. According to H', all coins have the same probability of landing heads. Its value is determined by fitting it to the entirety of outcomes of coin flips.

The question now is whether the best system entails $\mathrm{H}^{\prime}$ or $\mathrm{H}_{1}-\mathrm{H}_{2}$ ? The latter choice provides better fit because the probabilities are closer to corresponding relative frequencies of outcomes. But a system that entails $\mathrm{H}_{1}-\mathrm{H}_{2}$ rather than $\mathrm{H}^{\prime}$ will be less simple than one that entails H', as far as coins are concerned. This is because a system of the former type must in some way distinguish between the classes of coins and grant that they obtain their respective probability models. A system that assigns the same probability to every possible result of flipping a coin is simpler in this respect.

Consequently, the desiderata of simplicity and fit pull into different directions. To obtain the best system and the chances, we have to strike a balance between simplicity and fit. But it is not clear whether we should sacrifice simplicity to improve fit or the other way round. As mentioned above, Lewis does not specify a precise way to strike the balance, so we have to rely on our intuitions. At least my intuitions do not clearly favor H' over the set of $\mathrm{H}_{1}-\mathrm{H}_{2}$, nor the other way round, and I cannot discern another probability model that is clearly optimal. Overall, systems that entail $\mathrm{H}^{\prime}$ or $\mathrm{H}_{1}-\mathrm{H}_{2}$, respectively, 
seem to be roughly on par, as far as coins are concerned. Further, I don't see any rival hypotheses and any related rival system that is clearly better. The consequence is that there is no system that is overall best concerning coins and that there is no clear winner in the competition among the systems more generally. The last conclusion follows because every system has to take a stance concerning coins, and if there is a tie at this point, this will carry over to the overall comparison between systems. ${ }^{24}$

This argument goes through no matter of how exactly $\mathrm{H}^{\prime}$ or $\mathrm{H}_{1}-\mathrm{H}_{2}$ follow from the best system. It does not matter for fit concerning coin flips how $\mathrm{H}^{\prime}$ or $\mathrm{H}_{1}-\mathrm{H}_{2}$ follow from the best system. Further, every system that entails $\mathrm{H}_{1}-\mathrm{H}_{2}$ must at some point contain a more complicated probability model than a system that entails $\mathrm{H}^{\prime}$, or so at least it seems 25

If our assessments of the competing hypotheses and the respective systems are correct, then there is no clearly best system, and if there isn't, the identification of Humean chances is not straightforward any more. One possible answer is that there are no chances. Since there are SR probabilities in the world under consideration, we have SR probabilities that are not BS probabilities in a world that is close to ours, maybe even in our own world. The other option is to say that the chances are those probabilities upon which a potential plurality of best systems agree. In our example, $\mathrm{H}^{\prime}$ and $\mathrm{H}_{1}-\mathrm{H}_{2}$ may agree on some coins, but they cannot agree for both unbiased and our biased coins. Since both types of coins were assumed to carry SR probabilities, some SR probabilities do not coincide with BS probabilities once more ${ }^{26}$

This is not to say though that SR probabilities are never BS probabilities. In a world close to ours, in which there are lots of coins, but only unbiased ones, SR probabilities for coin flips turn out to be BS probabilities.

If there are BS probabilities for flips with a certain type of coin, an interesting question arises: What are the axioms in the best system from which these probabilities follow? One plausible answer is $\mathrm{H}$ or a generalization for symmetric bodies with $n$ equal faces, but there is, maybe, an alternative. The best system may contain a more detailed probability model for initial conditions of coin flips ${ }^{27}$ This model specifies a probability density in the phase space of initial conditions. ${ }^{28}$ Whether such a model is part of the best system

\footnotetext{
${ }^{24}$ The type of problem described in this paragraph is also pointed out by Frisch (2011), pp. 1004-1005 and by Beisbart (2014).

25 Frigg \& Hoefer (2010) argue that the best system contains a statement specifying the chances that certain symmetric solids with $n$ faces land on one of their faces (p. 359). They do not consider biased coins, dices etc. If biased coins count as regular solids, then my argument casts doubts on the idea that the rule given by Frigg \& Hoefer (2010) is part of the best system. If biased coins are not part of the regular solids covered by the rule, the question arises whether they have Humean chances.

${ }^{26}$ Some of these problems discussed here may be avoided if we allow for unsharp Humean chances (Glynn et al. 2014).

${ }_{27}$ Frigg \& Hoefer (2010), Sec. 4 suggest that, what is the best system according to their criteria, entails a probability rule of the outcomes of coin flips and, independently, a probability model for the initial conditions. Both probability models have to be roughly consistent with each other.

${ }^{28}$ By considering a probability density in phase space, we move to a continuous probability model. The definition of fit for such models has additional difficulties, see Elga (2004), particularly App. B. The argument in this paragraph relies on intuitive assessments of fit. It is thus presupposed that we can define a precise notion of fit that accords well with intuitive assessments of fit.
} 
depends on the world. In some worlds, the initial conditions of flips with our type of coin can be covered using a simple model with good fit. But in other worlds, this is not so. There are several competing probability models none of which is clearly optimal. In some worlds, women and men tend to produce significantly different initial conditions for coin flips. There then are two ways to model the empirical distribution of coin flips. Either we fit one probability model to the data, or we fit one for women and one for men. The latter model produces better fit, but is more complicated. Suppose again, that there is no clear winner, and that the probability models do not much overlap. In such worlds, we do not have BS probabilities for most initial conditions while there are BS probabilities for the outcomes. This accords well with the fact that there are SR probabilities for outcomes, but not for the initial conditions.

To sum up, SR and BS probabilities do not always coincide. SR probabilities do not cover probabilities that describe a stochastic dynamics, which are prime examples of BS probabilities in a world like ours. Conversely, SR probabilities are not always BS probabilities in all worlds.

\section{Two accounts, several problems}

In view of these differences, how should SR and BS be evaluated as accounts of ontic probabilities?

\subsection{Problems about Spielraum probabilities}

The most prominent problem about Spielraum probabilities is as follows (e.g. Rosenthal 2012, pp. 229-231). There is no conceptual link between the SR probability of a certain type of outcome and the actual frequencies with which this outcome arises in long series of trials. The numerical values of both may greatly differ. The reason is that the truth conditions SR are purely dynamic as shown above and do not restrict the initial conditions. Alone, these truth conditions do not imply the frequencies with which certain types of outcomes arise as a matter of fact.

To illustrate the problem, consider a world in which the same dynamic laws hold for coins as in our world, but in which the relative frequency with which most coins land heads differs greatly from the strike ratio of .5. For most coins, the frequency is rather, say, about .2. The reason is that, for most coins, initial conditions for patches leading to heads are less frequent than other initial conditions.

In this world, coin flips are described using the same phase space and the same Hamiltonian as in ours. Microconstancy with a strike ratio of .5 carries over to this world, and we obtain the same SR probabilities as in our world. Nevertheless, in this world, we would be reluctant to acknowledge SR probabilities as true chances. In fact, in this world, SR probabilities violate platitudes that we commonly associate with the notion of ontic probability: Chances are good predictors for frequencies; they can explain the values of frequencies, and, conversely, we can constrain the values of chances by using frequencies. All this is not true for this world any more. 
It may be objected that intuitions or platitudes about probabilities are not reliable and that they should be overridden in the example. This objection is not very convincing though because our intuitions can be justified using the Principal Principle (Lewis 1980). The latter spells out a very important idea about chances (cf. ibid., pp. 86-87), viz. that they should guide reasonable credences. In more detail, the principle reads as follows: If you think that, at $t$, a proposition $q$ has a chance of $r$, then you should set your degree of belief at that time at $r$ unless you have information about $q$ that goes beyond the chance of $q{ }^{29}$ Suppose now, for the sake of the hypothesis, that SR probabilities are real chances in our example world. Apply the Principal Principle. To do so, you must set your credence that a coin lands heads at .5. Accordingly, your credences for heads greatly differ from the corresponding frequencies. Although most coins land heads with a relative frequency of about 20\%, your credence that a coin lands heads is .5. As a consequence, you will often lose money if you bet on the outcomes of coin flips according to your credences. But this means that your credences aren't as reasonable as they could be. Reasonable credences, it seems, should enable us to be successful in betting. This at least is the central idea behind the consequentialist rationale that Hoefer (2007), pp. 580-587 proposes for the Principal Principle. Now if your credences aren't reasonable, then the SR probabilities can't be real chances in the world under consideration. This is support for our intuitive reluctance to call the SR probabilities chances in this world.

If SR probabilities fail to reflect the frequencies with which outcomes arise in some worlds, this has repercussions for the explanatory power of SR probabilities. Obviously, they don't explain frequencies in worlds in which the values of the frequencies significantly differ from those of the SR chances. It is further plausible to say that SR probabilities alone cannot even explain the values of frequencies in other, more "well-behaved" worlds either, because they are compatible with deviant frequencies. Other assumptions are needed to grant their explanatory power.

There are other types of examples in which certain SR probabilities clash with our intuitions. Suppose, for instance, that the coin flips in the universe can be ordered in natural way, e.g. according to cosmic time. Suppose further, it turns out that exactly every other coin flip yields heads. In such a world we may not be willing to assign probabilities to coin flips. The reason would be that the pattern of coin flips does not look random enough to deserve the predicate "chancy". Of course, the regularity in the outcomes of coin flips may be a matter of mere luck, in which case we would not object against there being chances about coin flips. But it is also possible that the regularity is not a matter of luck and that it can be explained. In this case, we would not be willing to assign chances to outcomes of coin flips, whereas SR would imply probabilities for them.

From a Humean perspective, the diagnosis for the problem is clear. The reason why the problem arises is that SR does not at all take into account the patterns of actual outcomes of coin flips. The problem is avoided under the Humean account because SR

29 The precise formulation of the Principal Principle is a matter of debate (see e.g. Lewis 1994, Hoefer 2007 pp. 552-553 and Frigg \& Hoefer 2010, pp. 356-357) and need not concern us here. 
refers to these patterns.

\subsection{Problems about best-system probabilities}

Turn now to BS. BS reflects the methods that working scientists use to obtain the values of chances: Scientists fit probabilistic models to data and thus to patterns in the real world. In doing so, they restrict themselves to simple probabilistic models; for instance, if possible, they adopt the assumption of probabilistic independence. And they only take these probabilities to be ontic if they don't find an alternative way to represent the information covered by the probabilities. In this respect, Lewis's account is correct.

There are problems though, which have already surfaced in our discussion. First, it is not clear whether fitting probabilities to the mosaic produces definite chances for outcomes of coin flips. It may well be that several incompatible probabilistic models can be fitted to the mosaic, none of them being clearly best (cf. our p. 15 above). If this is so, then there are no chances or at most those chances on which the models agree. This may not be enough, at least from an intuitive point of view, and we may want to conclude that Lewis's account is flawed $\$ 0$

Second, as already indicated, the BS account does not have much explanatory power. True, information about the frequencies of outcomes can be derived from corresponding BS probabilities, but the derivation seems hollow and uninformative at least in some cases because some BS probabilities are defined using those patterns they are supposed to explain. This is so for BS probabilities for outcomes of coin flips, if these probabilities are just part of the best system because the patterns of outcomes themselves call for a probability model, (cf. our discussion in Sec. 3). SR is more satisfying on this count because it picks certain dynamical characteristics of some systems, and the characteristics go some way towards providing an illuminating explanation why certain patterns arise ${ }^{31}$

In sum, we have found that both accounts suffer from problems, which are converse. SR has the potential to provide illuminating explanations of certain patterns, while BS does not. BS has a strong conceptual link to actual frequencies, while SR does not. It thus seems fruitful to combine BS and SR to arrive at a more convincing account of chances. The aim of the next section is to do so.

\footnotetext{
${ }^{30}$ Maybe, it is easier to obtain a robustly best system if we restrict ourselves to chances at a fundamental level as does Lewis. But the question then arises how we obtain chances of macroevents (cf. Hoefer 2007, p. 557 and Frigg \& Hoefer 2010, Sec. 4).

${ }^{31}$ Why is it a problem that BS has not much explanatory power? There are two possible reasons to require that an account of ontic probability provide illuminating explanations. First, if chances have considerable explanatory power, an account of them should capture this. Second, it is a reasonable demand that an account of ontic probabilities itself display explanatory power, even if chances do not explain much. If we are not just aiming at an analysis of meaning, it is sensible to require that the account explains well why probabilities are applied to some types of physical systems and not to others.
} 


\section{$5 \quad$ Playing with Spielraum and best system probabil- ities}

\subsection{Strengthening Spielraum probabilities}

SR identifies strike ratios with probabilities even in worlds in which the strike ratios do not match the values of corresponding frequencies. We must thus strengthen SR and tighten the connection between SR probabilities and the contingent frequencies with which outcomes in a world appear. The BS account of probabilities is more successful in linking probabilities and frequencies because BS probabilities are fixed using corresponding frequencies. Proponents of SR can learn from the BS account in this respect.

One way to strengthen SR is to restrict the frequencies with which the outcomes arise. But this is ad hoc, and the account would lose much of its explanatory power.

An alternative strategy is to restrict the actual frequencies with which the initial conditions originate in certain patches. This strategy is more promising because it is less ad hoc. Fairly weak restrictions on the initial conditions will suffice because the dynamics produces stable strike ratios independent of the precise distribution of the initial conditions (cf. the robustifying feature of SR probabilities; see our p. 8).

This strategy is adopted by Strevens (2011), p. 351 and Abrams (2012), particularly pp. 357-359 ${ }^{32}$ Following Strevens's lead, we demand that, typically, long, but finite series of initial conditions for coin flips follow a well-behaved empirical distribution in phase space. It can then be shown that the frequencies of outcomes of most long, but finite series of coin flips yield frequencies of outcomes that equal the corresponding strike ratios (up to a small margin of error; see Strevens 2003, Theorem 2.2 and Approximation 2.2.1, pp. 130-131).

Here are the details. We focus on a type of coin the dynamics of which is described using a specific phase space and a specific Hamiltonian. To define the probability that a coin of this type lands heads, we also consider all actual initial conditions for flips with our type of coin. For each coin flip, one initial condition is selected for a time $t_{f}-T_{\max }<t<t_{f}-T_{\min }$. The initial condition is not chosen by using the outcome of the flip. To select the initial condition per flip, we may e.g. use a pseudo-random generator to pick a time for every flip. We can only take one or a few initial conditions per coin flip because our series may otherwise contain initial conditions that are highly correlated with each other. We then consider all long, but finite natural series of actual initial conditions for coin flips. More about the length of a series will follow shortly. The series are called natural because they are identified in terms of natural characteristics. For instance, we group together those coin flips that occur in America between April and September 2012 to obtain one series. We do not allow for series that can only be identified in an unnatural, i.e., a very complicated manner. This is to exclude series that have been tailor-made by hand as to obtain a frequency of heads that significantly differs from the strike ratio (Strevens 2011, p. 352). Within each series, the order of

\footnotetext{
${ }^{32}$ See also Strevens (2003). For the purposes of this paper, I prefer Strevens (2011) to Strevens (2003) because it is more succinct regarding the problems discussed here.
} 
flips does not matter. Each series defines an empirical distribution of initial states. The empirical distribution is the set of initial states (points in phase space) within the series. It is not a probability distribution. It is useful though to represent the initial conditions of the series using a function defined on phase space. We partition each test region into even smaller regions, call them bins, that otherwise obey the same constraints as test regions. Their scale is such small that the dynamics would not be microconstant any more if the bins replaced the test regions. That is, there is a high fraction of bins that contain only one patch. For each bin, we take the fraction of initial conditions of this series within this bin. This yields a coarse-grained description of the empirical distribution; it is constructed by binning the data, as scientists put it. The requirement then is that, for each test region used to determine microconstancy, the bins in this test region have almost the same frequencies. Thus, the function defined by binning the initial conditions varies slowly within each test region, and in this sense, the distribution is wellbehaved. Following Strevens (2011), p. 352, we call an empirical distribution that obeys this condition macroperiodic. Note that macroperiodicity presupposes a breakdown of microconstancy at some small scale ${ }^{33}$

We can roughly understand why SR and macroperiodicity imply actual frequencies that approximate the strike ratios as follows: If the empirical distribution of initial conditions is macroperiodic, then most bins contain only one patch. However, the frequency of points in a bin varies very slowly as a function of position. Since the size of the bins is considerably smaller than that of the test regions, this is to say that, within most test regions, the frequency of actual initial conditions leading to heads is about the same and close to the strike ratio.

Macroperiodicity has to hold for almost all long series of actual coin flips. Only few exceptions are allowed. The series have to be long enough in the following sense: The bins have to be sufficiently full because, otherwise, the distribution cannot be well-behaved since the occupation of the neighboring bins will display significant variation simply due to the statistics of small numbers of trials. How long the series have to be turns on the scale for which microconstancy is not fulfilled any more. In our example of coin flips, the series have to be very long, and some 100 coin flips will certainly not suffice.

This is a problem because there are worlds in which a certain type of coin is not often flipped. The danger then is that macroperiodicity cannot even be tested for in these worlds and thus has no bite ${ }^{34}$ To alleviate the problem we can apply the following trick (Strevens 2011, pp. 361-362). To improve on the statistics of initial conditions in a world, we also take into account other types of coins. We group coins of several types and consider initial conditions with flips produced with coins of this very group taken

\footnotetext{
${ }^{33}$ Cf. Strevens 2003, Ch. 2, particularly Def. 2.5 on p. 129 for a more precise definition of macroperiodicity. Note though that my definition applies to empirical distributions directly, whereas the one by Strevens applies to probability densities, strictly speaking. There is another respect in which I slightly deviate from Strevens (2011). In his condition on empirical distributions (p. 352), Strevens also considers infinite series of trials and demands that, in this case, the limiting frequencies converge to a macroperiodic distribution. This is problematic because the limit of the frequencies depends on the order in which the points in phase space are considered. Cf. Hájek (2009), pp. 218-220. We need not consider infinite series because many of their finite sub-series are covered by the definition.

34 I'm grateful to an anonymous referee for drawing my attention to this point.
} 
together. This is of course only possible if the dynamics of all coins takes place in the same phase space. This condition is not too strong a requirement and is compatible with there being different types of dynamics, i.e., different Hamiltonians. Despite the different dynamics, the phase space may always be spanned by the same characteristics such as the angular velocity as taken by an observer at rest at the surface of the Earth. To simplify our discussion, I will assume that the larger group of coin flips comprises all coin flips.

To sum up, in general terms, the condition to be added on top of SR reads as follows:

$\mathrm{SR}+$ For almost all long, but finite natural series of trials on systems with the same phase space, the actual initial conditions of trials follow a macroperiodic distribution.

The proposal then is that SR and $\mathrm{SR}+$ are each necessary and jointly sufficient for chances. Probabilities that fulfill both SR and SR+ are called modified SR probabilities ${ }^{35}$

The strengthened Spielraum conception is immune against many counterexamples in which SR probabilities do not correctly predict frequencies. In worlds in which the SR probability of heads is .5 even though the frequencies of heads within long natural series of outcomes are typically .2 (cf. p. 17 above), $\mathrm{SR}+$ is violated. The empirical distributions over initial conditions cannot be macroperiodic in this case because, if they were, the frequencies would be about .5. Thus, according to SR and SR+, there are no chances for coin flips in this world. This is a plausible result, at least more plausible than are chances of .5 .

The other counterexample, in which there is a law-like regularity in the outcomes of coin flips, is also avoided (cf. p. 18 above). For suppose that a law-like regularity covers the outcomes of coin flips. The law connects the outcomes of coin flips with other characteristics, e.g. time or certain features of the coin. As a consequence, we can easily define a number of natural series of coin flips such that their relative frequency of heads is 1. Thus, the initial conditions of these series are not macroperiodic (otherwise, they would produce frequencies close to the strike ratio). Thus $\mathrm{SR}+$ is violated once more, and SR and SR+ imply the absence of chances, which is just what we'd like to say intuitively.

It is not the case though that $\mathrm{SR}+$ does not apply to all worlds in which the strike ratio for an outcome for a type of coin significantly differs from the corresponding frequency. First, SR + is vacuously true for worlds in which there are only few coin flips altogether. This is not a problem though because, if there are not many coin flips, we should be happy to admit the possibility that the probabilities do not reflect the frequencies. Second, SR+ can also hold in worlds in which the number of coin flips is large enough to allow for a meaningful test of macroperiodicity, but in which each type of coin is only flipped

\footnotetext{
35 The notion of macroperiodicity is here defined on the basis of binning data. It may be suggested that the data in long, but finite series of coin flips rather be fitted using simple probability models. We may then demand that the best fitting model varies only slowly. This would well accord with ideas on which BS is built. It is doubtful though whether the alternative suggestion works. Fitting a simple probability model to the data leads to a model that is smooth and varies slowly independently of whether the empirical distribution of initial conditions is macroperiodic.

Something like SR+ is already anticipated by von Kries (1886), e.g. pp. 50-51.
} 
rarely. In this world, for some types of coins, the strike ratios can greatly differ from the frequencies of outcomes. Again, I think this is not too big a problem from the intuitive point of view because the problem concerns probabilities that are not instantiated very often.

Turn now to the explanatory power of our new account of ontic probability. SR and $\mathrm{SR}+$ do not just describe the frequencies with which outcomes arise in our world, but also explain them. SR and SR+ entail that most long series of coin flips display a certain statistics in their outcomes. Since SR holds due to laws, the derivation has explanatory power. The derivation can be extended to small series, if an additional assumption is made, see Strevens (2011), pp. 353-354. The additional assumption needed is that the distribution of those natural subsequences of a certain length that are part of a sequence is also macroperiodic in a well-defined sense. This assumption and SR lead to probabilistic independence (ibid.). Some additional assumption is needed because we cannot expect that the frequencies of outcomes within most small series match the respective probabilities if the flips strongly depend on each other probabilistically.

We are now in a position to render more precise what has been said about the explanatory power of SR. At first sight, SR seems to explain why E1 and E2 defined above hold. The idea is just that the microconstant dynamics leads to E1 and E2 (see p. 12 above). But as counterexamples make manifest, SR on itself can't be the whole explanation of the frequencies because it doesn't imply the observed frequencies in some worlds (see p. 18 above). SR+ excludes many counterexamples, and thus SR and SR+ provide a full explanation in a lot of worlds. It is quite natural to forget about $\mathrm{SR}+$ because it is a natural expectation that the initial conditions are not fine-tuned, but rather follow the Lebesgue measure.

Strevens (2011) does not rest content with SR and SR+. He demands that SR+ holds not only in our world, but also counterfactually, i.e., in most possible worlds close to ours (ibid., p. 359). Strevens's reason is that he takes probabilities to explain nomic frequencies, i.e., frequencies that are counterfactually robust. This strengthening of SR+ comes with costs though (cf. Rosenthal 2012, p. 232). SR+ can only hold in most possible worlds close to ours if we have a measure over worlds. Strevens suggest that this measure is derived from well-known measures over physical characteristics, e.g. time, space etc. (Strevens 2011, p. 258).

In the following, I will concentrate on SR and SR+ and neglect counterfactual robustness of SR+. My reasons to do so are mainly pragmatic: We can make some progress concerning the problems mentioned above in Sec. 4 without thinking about counterfactual robustness.

\subsection{Discussion points}

Modified SR + probabilities seem to be on firm ground, then. Nevertheless, there are possible objections. Rosenthal (2012), pp. 231-234 criticizes the accounts proposed by Strevens (2011) and Abrams (2012) for three reasons and, if successful, his criticisms carry over to the combination of SR and SR+. In the following, I'll discuss Rosenthal's objections as if they were leveled against the combination of SR and SR+. 
Rosenthal's first criticism is that $\mathrm{SR}+$ requires a certain distribution over initial conditions. Rosenthal fears that reference to a distribution renders the proposal circular or uninformative as an account of ontic probability (ibid., p. 232).

This criticism is not convincing though. To begin with, we have to distinguish between probability models and what I have called empirical distributions. The former specify for a range of propositions, while the latter comprise data points and are not probabilities. We can think of an empirical distribution as a collection (i.e., a set) of particular instances of some type of event. Typically, the instances scatter with respect to the values of some characteristics, e.g. momentum. This leads to a certain distribution of momentum in this set of instances. This distribution can well be represented by defining suitable bins in momentum space and counting the instances per bin. The binned distribution can then be shown as a histogram. There is no need here to talk about probabilities. Neither the empirical distribution nor the distribution of a certain characteristics within this set involves probabilities. Thus, SR+ does not lead to circularity. ${ }^{36}$

It may be objected that $\mathrm{SR}+$ does go beyond empirical distributions qua collections of data points because the data points are used to construct a function $f$ that maps bins in phase space to real numbers. This function, which is referred to in my definition of macroperiodicity, can be seen as a probability density. It may thus be objected that $\mathrm{SR}+$ does define a probability model and so renders the account circular. This objection is not convincing though. The function $f$ may be interpreted as a probability model, but it need not. And even if $f$ is interpreted as a probability density, this would not render the account circular. For the probability would be purely formal. It would be a probability density in the sense of mathematics. It is not required by $\mathrm{SR}+$ that resulting probabilities are chances or ontic probabilities. But our task is to provide truth conditions for assignments of chances, and not of formal probabilities, which are needed in any case. We can thus use formal probabilities to define chances. In a similar way, Lewis's account of chances draws on formal probabilities to define chances.

Rosenthal's second objection is as follows (ibid., pp. 233-234). SR identifies chances with strike ratios. The strike ratios in turn are defined using a measure over phase space, viz. the Lebesgue measure. But there are other measures over phase space. Under such measures, some regions in phase space obtain significantly more weight than they have under the Lebesgue measure, or the other way round. Thus, the dynamics of a system may be microconstant with respect to one measure, but not with respect to another, or the numerical value of the strike ratio may depend on the choice of the measure. This may lead to the following problems: a. If the Lebesgue measure is chosen, SR and SR+ imply chances for coin flips, but SR is violated if a different measure is assumed, such that there are no chances for coin flips any more. b. SR is violated for a certain system

\footnotetext{
36 The distinction between empirical distributions and probability models is often neglected for two reasons. First, the latter are sometimes called probability distributions. Second, actual data are often conveniently described in terms of a probability model that fits the data well. It is then said that the data are normally distributed, for instance. Nevertheless, there is a distinction between empirical distributions and probability models, and it is not ad hoc. When statisticians say that they fit a probability model to the actual data, they implicitly refer to the distinction.
} 
assuming the Lebesgue measure, but if we construct a different measure by hand, SR and SR+ are fulfilled and we obtain chances relatively to this measure. c. SR and SR+ are fulfilled under two measures for a system, but each measure leads to different values of the chances.

Further, to define phase space and to identify suitable small test regions, we use certain physical characteristics (e.g. position and momentum). Again, there are alternative choices that will lead to different test regions because regions that are small in some characteristics are not small in others. Consequently, the dynamics of a system may be microconstant with respect to one choice of variables, but not with respect to another, or the strike ratio may depend on the variables chosen. Rosenthal (2012), p. 234 concludes that "[w]ithout an initial-state space with definite proportions of the various outcomes the range [Spielraum] conception does not get off the ground."37

It is certainly right that the Spielraum conception of probabilities assumes a specific measure and specific characteristics to define the test regions. But why is this a problem? Rosenthal's worry seems to be this: Since there are alternative choices that imply markedly different claims about probabilities under SR, we have to justify our choices. The worry then is that there is no way to justify the choices objectively.

I do not agree with these worries for two reasons.

First, certain choices of measures and variables can be justified. Begin with the Lebesgue measure. It is distinguished by the fact that it is preserved under the dynamics: Pick a region in phase space and measure its Lebesgue volume. Let the points of this region follow the dynamics of the system for a time interval $\Delta t$. The Lebesgue volume of the region of the evolved points is the same as the original one ${ }^{38}$

The conservation of Lebesgue volume is in fact essential for SR probabilities. If the Lebesgue volume was not preserved, then we might obtain different values of SR probabilities for different times $t_{f}-T_{1}, t_{f}-T_{2}$ for $T_{1} \neq T_{2}>T_{\min }$. But this is not what we want.

Turn now to the choice of the variables and to the identification of the test regions. To begin, note first that SR is not the only condition for there being probabilities. SR+ has to hold too, and it implicitly restricts the choice of the test regions. The distribution over initial conditions has to be macroperiodic, and macroperiodicity will often be lost if we switch to different variables and thus to different test regions. See Strevens (2003), Sec. 2.5 for more discussion of this point.

Note further that Hamiltonian dynamics distinguishes certain coordinates. The Hamiltonian equations hold only in so-called canonical coordinates, and these equations are in turn distinguished by their simplicity. Further, the Lebesgue measure is form-invariant under all canonical coordinates. In canonical coordinates, an infinitesi-

\footnotetext{
37 In the following, I do not distinguish between physical characteristics and coordinates. Even though there is a distinction, a switch between different coordinates may be seen as a switch between two physical characteristics. I also sometimes call the characteristics variables.

38 This is Liouville's Theorem again. It does not exclude though other measures that are preserved by the dynamics. Nevertheless, generally, other measures will not be preserved by the dynamics. We will presently argue for the choice of certain variables, and once we have chosen these variables, we are only interested in measures that are simple to express in these variables, which should be sufficient to fix the measure.
} 
mal volume element can be expressed as a product of infinitesimal coordinate differences Arnol'd 1989, Sec. 9.1) ${ }^{39}$ So there seem to be good reasons to choose canonical variables.

Nevertheless, different sets of canonical coordinates do lead to different test regions. The effect can be that the dynamics is microconstant in some canonical variables, but not in others. So even within the Hamiltonian formalism, there is some arbitrariness here. However, there are other reasons to pick some coordinates rather than others. Characteristics such as space and coordinates that leave distances invariant are natural in that they lead to simple formulations of the physical laws. Why distort, say, momentum and switch to momentum ${ }^{\star}$ if the laws of physics look much more complicated if formulated in terms of momentum ${ }^{\star}$ ?

In sum, there are good reasons to choose some measure and some coordinates rather than others. A common thread in the justification of the measure and the coordinates is simplicity. It may be objected that appeals to simplicity do not suffice at this point, because simplicity is a pragmatic factor that depends on our own interests. But such an objection would be hypocritical. Natural scientists appeal to considerations of simplicity in their investigations too, but many people are nevertheless prepared to accept wellsupported findings of natural science as objective truths 40

Strevens (2003), pp. 88-96 provides a different argument to justify the choice of some coordinates (cf. also Abrams 2012, p. 363-366). The basic idea is as follows: SR+ requires macroperiodicity of the empirical distribution of the initial conditions. As already mentioned on p. 26, this constrains the choice of the coordinates. If macroperiodicity is additionally supposed to be stable under perturbations that tend to affect the initial conditions, the choice of coordinates is even more restricted, and only the usual coordinates are left. I agree with Rosenthal (2012), p. 234 that this doesn't provide a convincing justification of some coordinates because Strevens has to make assumptions as to what perturbations tend to be like.

The second reason why I reject Rosenthal's second objection is as follows. Our task is to provide an account of certain probabilistic statements. We have to explain what speakers refer to if they utter probabilistic statements. We do so by providing truth conditions for the statements. These truth conditions can refer to some measure and coordinates rather than to others. There is no need to justify the choice of the measure and the coordinates if only we specify what people refer to or express.

Compare this to the following example. Suppose, our task is to identify what French speakers mean when they utter the word "je". At some point, we discover that "je" serves as a pointer to the speaker. Can we object that the choice of the speaker is arbitrary (there are other people, after all!)? This objection would not make sense. If a French speaker uses the word "je", she has decided to refer to herself, and this is what an investigation of "je" has to make plain. The point carries over to a conceptual analysis of "chance" or "ontic probability".

\footnotetext{
39 This is another way in which the Lebesgue measure is distinguished.

40 Note also that Lewis uses considerations of simplicity to analyze chances. There are of course problems if simplicity is too vague or if people disagree on what is simple. I do not think that we run into this problem here.
} 
Admittedly, our case is not entirely analogous because we are not so much interested in what people actually mean if they ascribe a probability to a proposition. Rather, we are seeking after truth conditions that imply what people want to imply if they talk about chances. That is, the truth conditions should express what speakers may sensibly mean if they talk probabilistically. But again, this explanation may refer to some measure and coordinates rather than to others. There is no need to justify the choice of some variables, at least as long as the truth conditions provided imply those kinds of things that people imply in probabilistic talk 41

But maybe this is too quick. Maybe we have overlooked something that needs justification. SR and SR+ use natural variables, and they furnish an account of ontic probabilities. A question then is: Why do we implicitly refer to these variables when we talk of probabilities? To appreciate the point of this question, consider the possibility of a type of physical system in which SR and SR+ do not hold relatively to natural coordinates, but in which they do so relatively to some unnatural coordinates. Why do we withhold probabilistic claims with respect to the latter systems, while we accept ontic probabilities about coin flips?

Presumably the reason is as follows: We (scientists or not) use measures and coordinates that make things simple all the way down, not just when we talk about probabilities. So why not refer to them if we are concerned with probabilities? The deeper reason may be this. Scientists have the task to devise a good or the best system in the sense of Lewis. The question then only is whether we find a simple pattern using measures and coordinates that make things simple. A pattern that is simple with respect to measures or coordinates that render things very complicated otherwise will certainly not be part of the best system.

This is not meant to imply that our notion of probability is subjective. The truth conditions for probability assignments are objective. The point is only this: The explanation of why we apply the concept of probability to one case, but not to another turns on ourselves and our purposes.

A yet different question about justification is why we are entitled to assume that $\mathrm{SR}$ and $\mathrm{SR}+$ is fulfilled in some cases, e.g. regarding coin flips? This assumption needs an empirical, inductive justification. As far as our evidence so far is concerned, the assumption holds true. It is thus reasonable to project it to other cases, notably the future. See North (2010) for a forceful defense of this move.

Rosenthal's third objection is as follows (Rosenthal 2012, p. 234). We expect that accounts of ontic probabilities provide means to explain why outcomes arise with certain frequencies. But SR + is a constraint on actual frequencies, and this prohibits an illuminating explanation of the patterns within the outcomes, or so the objection goes.

\footnotetext{
${ }^{41}$ Strevens (2011), p. 351 contains a different reply to the objection that the notion of SR probabilities turns on an arbitrary choice of a measure: He only requires that there be a measure with respect to which microconstancy and macroperiodicity are fulfilled. I do not think that this will do. Suppose that microconstancy and macroperiodicity hold with respect to some very unnatural measure, but not with respect to other, more natural variables. Suppose further that, in the natural variables, the initial conditions leading to the $o_{i}$ could be described very easily. That is, the patches do not display the geometry that we expect in a well-mixed dough. I doubt whether we would allow for chances in this case.
} 
True, SR+ is not about the frequencies of outcomes, but rather about the frequencies with which certain initial conditions arise in series of trials; nevertheless, granted determinism, frequencies of initial conditions translate into frequencies of outcomes.

To see the problem more clearly, consider the way in which SR and SR+ explain the statistics of coin flips. SR implies that there is a deterministic dynamics such that the statistics of coin flips can be traced back to statistics for the occupation of patches, which have a very peculiar geometry. Thus, the statistics of the outcomes is the same as the statistics of the patches. SR+ in turn implies that the statistics of the patches is such that we can ascribe probabilities to them and thus to the outcomes. This explanation seems shallow and superficial. Facts about some statistics are traced back to facts about another statistics at an earlier instance of time. No explanation is given of why we obtain a certain statistics for coin flips and for patches to begin with, or so the objection goes.

I do not agree with this criticism, at least not fully. Note first that most explanations of phenomena draw on matters of contingent facts that concern the history of the systems under consideration ${ }^{42}$ For instance, to explain why a particular system is now in state $s_{1}$, we can specify the dynamics of the system and the state in which it was at an earlier time. Information of the latter sort is in some sense of the same kind as is the information that the phenomenon to be explained occurs now. We have nevertheless a good explanation.

Further, the condition of SR + on the statistics of the initial conditions is very weak in some respect. SR+ only requires that almost all long natural series of initial conditions display a macroperiodic empirical distribution. This is compatible with different empirical distributions in different series of initial conditions. Further, SR+ alone does not explain why we observe stable frequencies for the outcomes. Nor does macroperiodicity imply the value of the frequency with which an outcome arises in long series of flips. This value is explained by the value of the strike ratio which is a feature of the dynamics, and thus in a way law-like 43 In sum, important features of the statistics of the outcomes are not put in by hand via $\mathrm{SR}+$, as it were; they rather only follow if the dynamics is taken into account (cf. Abrams 2012, p. 366).

Despite all this, it is true that $\mathrm{SR}+$ refers to empirical distributions in the real world, and this rather weak assumption is pivotal for deriving the statistics of outcomes we observe in our world. It may thus be suggested that real chances must offer a stronger explanation of the statistics of outcomes than do SR and SR+. This suggestion may be supported with the following claim: The frequencies with which we observe outcomes of coin flips in series are not just a matter of contingent fact, but rather counterfactually robust (Strevens 2011, pp. 341-342).

I am not sure whether this part of the objection stands. It raises larger issues about explanation and counterfactual robustness that are beyond the scope of this paper. But if it stands, then we can modify SR + to avoid the objection (see in particular Strevens 2011). The obvious idea is that $\mathrm{SR}+$ is not just actually true, but that it also tends to be true or that there are certain facts that tend to make $\mathrm{SR}+$ true (to use a formulation by Rosenthal 2012, p. 232). Of course, these facts must be facts about other possible

\footnotetext{
${ }^{42}$ See e.g. Hempel \& Oppenheim (1948), pp. 135-140.

${ }^{43}$ See Abrams (2012) p. 366 for the point about the value of the strike ratio.
} 
worlds. More precisely, most close possible worlds must obey SR+.

This modification of SR+ grants counterfactual robustness, but it is very speculative because it refers to other possible worlds. The obvious question is why we are entitled to assume the required facts about close possible worlds (cf. Rosenthal 2012, p. 232). Taking up a suggestion by Strevens (2011), p. 355, we can perhaps answer this question as follows: Facts that support SR+ concerning our own world also support that SR+ holds within most possible worlds close to ours (cf. also Abrams 2012, p. 366).

In sum, I do not think that Rosenthal's objections, if leveled against SR and SR+, are successful.

\section{Conclusions}

Where does this leave us? Spielraum probabilities as defined by SR above apply to the outcomes of certain processes such as coin flips. These processes are characterized by a microconstant dynamics. Their outcomes can be traced back to patches in a phase space of initial conditions, where the patches display a peculiar pattern: Relative to the Lebesgue measure, the regions from a suitable partition of phase space each contain the same share of a particular patch. This share defines the strike ratio, which is taken to be the probability.

This account of probability is limited in scope because it requires a special dynamics. Nevertheless, its scope includes a number of classical (i.e., non-quantum) gambling devices. A strength of the account is that it goes some way to explaining why the outcomes of coin flips, though difficult to predict individually, display certain stable features in their empirical distribution.

By contrast, best system probabilities as proposed by Lewis do not have a principled restriction in scope. We expect them wherever patterns of actual events display stable statistical features that escape a non-probabilistic description in terms of deterministic laws. This limits the explanatory power of best system probabilities. Even though some such probabilities can be explained using others and the laws of nature, other best system probabilities are simply effective means of summarizing the patterns found in the mosaic.

Since the outcomes of coin flips display patterns that lend themselves to a probabilistic model, it seems as if there are best system probabilities of such flips. If this is so, then at least some Spielraum probabilities are at the same time Humean chances. But I have argued that things are not as simple as that. First, there are only best system probabilities if there is a unique best system including the probabilities or if a number of best systems agree on them. The uniqueness is a problem because there are rival ways of setting chances on macro-events such as coins landing heads. The rivals are not easily comparable because the criteria of fit and simplicity pull into different directions. Second, Spielraum probabilities only turn out to be Humean chances if the Spielraum probabilities roughly agree with the respective frequencies of outcomes. This need not be so since we can think of worlds in which this is not so. Even if coins land heads with a Spielraum probability of $50 \%$, it is possible that we obtain $20 \%$ heads when we flip coins. This is so if the initial conditions do not sample the patches according to their 
Lebesgue measures. As a consequence, Spielraum probabilities do not fully explain the statistics of outcomes of coin flips etc.

This is clearly a problem about Spielraum probabilities. To solve this problem, a conceptual link with the frequencies with which outcomes arise as a matter of fact is required, as we have it for best system probabilities. We have thus strengthened Spielraum probabilities by restricting the frequencies with which initial conditions arise. In detail, most long series of initial conditions must follow a macroperiodic distribution. This move is suggested by Strevens (2011) and Abrams (2012). The account that arises can stand a number of objections. The probabilities grant the right kind of frequencies in long series of trials, and further furnish a non-trivial explanation of frequencies. Maybe, however, the explanation is still too weak because the statistics of outcomes is more than contingent, whereas the new condition holds only contingently.

To conclude, let us suppose that the strengthened Spielraum probabilities provide a defensible account of ontic probability and let us turn to the broader picture. What ontic probabilities are there and which of them are modified SR probabilities?

Since the Spielraum probabilities have been restricted even more, they are still unable to account for quantum mechanical probabilities. The latter may be interpreted as Humean best system probabilities. Perhaps, there is a more powerful way of thinking of quantum mechanical probabilities, but they should at least turn out to meet the criteria of best system probabilities. The consequence is that there are at least two types of ontic probabilities, viz. modified Spielraum probabilities and best system probabilities. Nevertheless, some probabilities may be categorized as both modified SR and BS probabilities.

Is this a sensible picture, at least for worlds close to ours? The answer turns on the question of how both types of probabilities relate. Due to the problems about constructing a uniquely best system probability model covering macrochances, the answer to the latter question is not clear. There are two possibilities: Under the first, there is a uniquely best system that roughly reproduces Spielraum probabilities. This will not be the case in all worlds, but may be the case in some worlds like ours (including our own). Then modified Spielraum probabilities are one way of realizing best system probabilities. The latter form an umbrella term of probability, and strengthened Spielraum probabilities are covered by it. If the best system comprises a model for the initial conditions too, then the whole Spielraum story is part of the best system. If not, then it provides complementary information that accounts for the statistics within certain patterns in a more illuminating way.

The second possibility is that Spielraum probabilities are to a large extent not matched by best system probabilities because the best system does not assign any probabilities to macroevents such as coin flips. In this case, modified Spielraum probabilities are a complement to best system probabilities that seems urgently called for from an intuitive point of view. After all, probabilities of the outcomes of gambling devices are prime examples of ontic probabilities. If things are such as the second possibility has it, there is a certain division of labor between both kinds of probabilities. Whereas Spielraum probabilities arise at the macrolevel due to a certain type of dynamics, best system probabilities emerge at the fundamental level and cannot be further explained. 
The story would have to be complemented by saying something about probabilities postulated within statistical mechanics, genetics and so on, but this is something for a new project.

\section{Acknowledgments}

I'm very grateful for detailed and valuable criticism by two anonymous referees and for comments by the participants of the workshop at Bonn. Thanks also to Jacob Rosenthal and Carsten Seck for the invitation to contribute this paper and for comments on the paper. I'm also grateful to Joannes Campell, Martina Jakob and Lukas Lüscher for proofreading.

\section{References}

Abrams, M., Mechanistic probability, Synthese 187 (2012), no. 2, 343-375.

Arnol'd, V. I., Mathematical methods of classical mechanics, Springer, New York etc., 1989.

Beisbart, C., Good just isn't good enough - Humean chances and Boltzmannian statistical physics, in: New Directions in the Philosophy of Science (Heidelberg) (Hartmann, S., Weber, M., Gonzalez, W., Dieks, D., \& Uebel, T., eds.), The Philosophy of Science in a European Perspective, Springer, Cham etc., pp. 511-529.

Briggs, R., The metaphysics of chance, Philosophy Compass 5 (2010), no. 11, 938-952.

Diaconis, P., Holmes, S., \& Montgomery, R., Dynamical bias in the coin toss, SIAM Review 49 (2007), no. 2, pp. 211-235 (English).

Diaconis, P., A place for philosophy? The rise of modeling in statistical science, Q. Appl. Math. LVI (1998), no. 4, 797-805.

Elga, A., Infinitesimal chances and the laws of nature, Australasian Journal of Philosophy $\mathbf{8 2}$ (2004), 67-76.

Frigg, R. \& Hoefer, C., Determinism and chance from a Humean perspective, in: The Present Situation in the Philosophy of Science. (Dieks, D., Gonzalez, W., Hartmann, S., Weber, M., Stadler, F., \& Uebel, T., eds.), Springer, Berlin and New York, 2010, pp. 351-37.

Frigg, R. \& Hoefer, C., The best Humean system for statistical mechanics, manuscript, 2012.

Frisch, M., From Arbuthnot to Boltzmann: The past hypothesis, the best system, and the special sciences, Philosophy of Science 78 (2011), 1001-1011.

Gillies, D., Philosophical Theories of Probability, Routledge, London and New York, 2000.

Dardashti, R., Glynn, L. F., Thebault, K. \& Frisch, M., Unsharp Humean chances in statistical physics: A reply to Beisbart, in: New Directions in the Philosophy of Science (Heidelberg) (Hartmann, S., Weber, M., Gonzalez, W., Dieks, D., \& Uebel, T., eds.), The Philosophy of Science in a European Perspective, Springer, Cham etc., 2014, pp. 531-542.

Hájek, A., 'Mises redux'-redux. Fifteen arguments against finite frequentism, Erkenntnis $\mathbf{4 5}$ (1997), 209-227.

Hájek, A., Fifteen arguments against hypothetical frequentism, Erkenntnis 70 (2009), 211-235.

Hájek, A., Interpretations of probability, in: The Stanford Encyclopedia of Philosophy (Zalta, E. N., ed.), spring 2010 ed., 2010, http://plato.stanford.edu/archives/spr2010/entries/probability-interpret/. 
Heidelberger, M., Origins of the logical theory of probability: Von Kries, Wittgenstein, Waismann, International Studies in the Philosophy of Science 15 (2001), no. 2, 177-188.

Hempel, C. G. \& Oppenheim, P., Studies in the logic of explanation, Philosophy of Science $\mathbf{1 5}$ (1948), 135-175.

Hoefer, C., The third way on objective probability: A sceptic's guide to objective chance, Mind 116 (2007), 549-596.

Hopf, E., On causality, statistics and probability, Journal of Mathematics and Physics 13 (1934), 51-102.

Hopf, E., Über die Bedeutung der willkürlichen Funktionen für die Wahrscheinlichkeitstheorie, Jahresbericht der Deutschen Mathematiker-Vereinigung 46/I (1936), 179-194.

Howson, C. \& Urbach, P., Scientific reasoning: The Bayesian approach, third ed., La Salle, Open Court, 2006.

Kamlah, A., Probability as a quasi-theoretical concept - J.v. Kries' sophisticated account after a century, Erkenntnis 19 (1983), no. 1-3, 239-251.

Keller, J. B., The probability of heads, The American Mathematical Monthly 93 (1986), no. 3, pp. 191-197 (English).

Lewis, D., A subjectivist's guide to objective chance, in: Studies in Inductive Logic and Probability. Vol II (Jeffrey, R. C., ed.), University of California Press, Berkeley, 1980, here quoted from the reprint in Lewis (1986b), pp. 84-113.

Lewis, D., Introduction, in: Philosophical papers. Volume II, Oxford University Press, New York, 1986, pp. ix-xvii.

Lewis, D., Philosophical papers. Volume II, Oxford University Press, New York, 1986.

Lewis, D., Postscripts to "A subjectivist's guide to objective chance", in: Philosophical Papers. Vol II (Lewis, D., ed.), Oxford University Press, New York, 1986, pp. 114-132.

Lewis, D., Humean supervenience debugged, Mind 103 (1994), 473-490, reprinted in Lewis, D. (1999), Papers in Metaphysics and Epistemology, Cambridge University Press, Cambridge.

Loewer, B., Determinism and chance, Studies in History and Philosophy of Modern Physics 32 (2001), 609-620.

Meacham, C. J. G., Contemporary approaches to statistical mechanical probabilities: A critical commentary - Part I: The indifference approach, Philosophy Compass 5 (2010), no. $12,1116-1126$.

Meacham, C. J. G., Contemporary approaches to statistical mechanical probabilities: A critical commentary - Part II: The regularity approach, Philosophy Compass 5 (2010), no. $12,1127-1136$.

Myrvold, W. C., Deterministic laws and epistemic chances, in: Probability in Physics (Ben-Menahem, Y. \& Hemmo, M., eds.), The Frontiers Collection, Springer, Berlin and Heidelberg, 2012, pp. 73-85 (English).

North, J., An empirical approach to symmetry and probability, Studies in History and Philosophy of Science Part B 41 (2010), no. 1, 27-40.

Poincaré, H., Calcul des probabilités, G. Carré, Paris 1896, second edition Gauthier-Villars, Paris 1912.

Rosenthal, J., The natural-range conception of probability, in: Time, Chance, and Reduction. Philosophical Aspects of Statistical Mechanics (Ernst, G. \& Hüttemann, A., eds.), Cambridge University Press, Cambridge, 2010, pp. 71-91.

Rosenthal, J., Probabilities as ratios of ranges in initial-state spaces, Journal of Logic, Language, and Information 21 (2012), 217-236. 
Strevens, M., Bigger than chaos. Understanding complexity through probability, Harvard University Press, Cambridge (MA), 2003.

Strevens, M., Probability out of determinism, in: Probabilities in Physics (Beisbart, C. \& Hartmann, S., eds.), Oxford University Press, Oxford, 2011, pp. 337-362.

von Kries, J., Die Principien der Wahrscheinlichkeitsrechnung: Eine logische Untersuchung,

J. C. B. Mohr, Freiburg i.B., 1886, second edition J.C. B. Mohr: Tübingen 1927.

von Plato, J., The method of arbitrary functions, British Journal for the Philosophy of Science 34 (1983), no. 1, 37-47. 